\title{
Diversity of endophytes across the soil-plant continuum for Atriplex spp. in arid environments
}

\author{
Mohammad E TAHTAMOUNI ${ }^{1}$, Sa’eb KHRESAT ${ }^{2}$, Mary LUCERO ${ }^{1}$, Jesus SIGALA ${ }^{1,3}$, \\ Adrian $\mathrm{UNC}^{1,4,5^{*}}$ \\ ${ }^{1}$ New Mexico State University, Department of Plant \& Environmental Sciences, Las Cruces NM 88003, USA; \\ ${ }^{2}$ Jordan University of Science and Technology, Department of Natural Resources and Environment, Irbid 22110, Jordan; \\ ${ }^{3}$ Seco Spice, Chamberino NM 88027, USA; \\ ${ }^{4}$ Boreal Ecosystems Research Initiative, Memorial University of Newfoundland, Corner Brook A2H 5G4, Canada; \\ ${ }^{5}$ University of Leeds, School of Geography, Leeds LS2 9JT, UK
}

\begin{abstract}
Endophytes are hypothesized to be transferred across the soil-plant continuum, suggesting both the transfers of endophytes from environment to plant and from plant to soil. To verify this hypothesis and to assess the role of locality, we evaluated the similarity of microbial communities commonly found both in soils and endophytic communities in three arid regions, i.e. the Jornada LTER (Long Term Ecological Research) site in New Mexico, USA, and the research station of Jordan University of Science and Technology (JUST) and Khanasri research station in Badia region of Jordan. Rhizosphere and non-rhizosphere soils, leaves and seeds of Atriplex spp. were sampled. Diversity and distribution of bacteria and fungi across the soil-plant continuums were assessed by tag-encoded FLX amplicon pyrosequencing and sequence alignment. Of the total bacterial OTUs (operational taxonomic units), $0.17 \%$ in Khanasri research station, $0.16 \%$ in research station of JUST, and $0.42 \%$ in Jornada LTER site were commonly found across all the plant and soil compartments. The same was true for fungi in two regions, i.e. $1.56 \%$ in research station of JUST and $0.86 \%$ in Jornada LTER site. However, in Khanasri research station, $12.08 \%$ of total fungi OTUs were found in at least one soil compartment and one plant compartment. Putative Arthrobacter, Sporosarcina, Cladosporium and members of Proteobacteria and Actinobacteria were found across all the soil-plant continuums. Ascomycota, mainly including Didymellaceae, Pleosporaceae and Davidiellaceae were present across all the soil-plant continuums. Microbial communities in two regions of Jordan were similar to each other, but both of them were different from the Jornada LTER site of USA. SIMPER (similarity percentage) analysis of bacterial and fungal taxa for both soil and endophyte communities revealed that dissimilarities of two bacterial genera (Arthrobacter and Sporosarcina) and two fungal genera (Cladosporium and Alternaria) are very high, so they play key roles in the soil-plant continuums. A weighed Pearson correlation analysis for the specific bacterial OTUs in the soil-plant continuums only showed high similarity between the two regions of Jordan. However, fungal groups showed higher similarities among all regions. This research supports the hypothesis of continuity of certain bacterial and fungal communities across the soil-plant continuums, and also explores the influences of plant species and geographic specificity on diversity and distribution of bacteria and fungi.
\end{abstract}

Keywords: endophytes; microbial diversities; soil-plant continuum; pyrosequencing; Atriplex spp.

Citation: Mohammad E TAHTAMOUNI, Sa'eb KHRESAT, Mary LUCERO, Jesus SIGALA, Adrian UNC. 2016. Diversity of endophytes across the soil-plant continuum for Atriplex spp. in arid environments. Journal of Arid Land, 8(2): 241-253. doi: 10.1007/s40333-015-0061-9

*Corresponding author: Adrian UNC (E-mail: aunc@grenfell.mun.ca)
Received 2015-04-25; revised 2015-10-09; accepted 2015-10-26
(C) Xinjiang Institute of Ecology and Geography, Chinese Academy of Sciences, Science Press and Springer-Verlag Berlin Heidelberg 2016 
According to the widely accepted definition of endophytes proposed by Wilson (1995), endophytes are microorganisms that exist within plant tissues, or spend some phase of their life cycle inside living plant tissues but do not harm the plant. They could be commensals or mutualists, or occasionally borderline pathogenic but with no symptoms exhibited by the host. All plant species are believed to harbor endophytes. Many endophytes are shared and transferred among plants, horizontally (Saikkonen et al., 2004; Gallery et al., 2007); and many of them are seedborne, transferring vertically from generation to generation and influencing the seed germination and plant fitness (Barrow et al., 1997, 2004; Schardl et al., 2004; Lucero et al., 2008, 2011; Dalling et al., 2011; Johnston-Monje and Raizada, 2012), offering resistance to pathogens or minimizing herbivory (Doty, 2008). Their specificity to hosts or environments varies among endophyte taxa (Leuchtmann, 1993).

Soil microorganisms migrate towards the area surrounding the plant roots, namely the rhizosphere, and colonize the area immediately in response to root exudates (Lynch and Whipps, 1991; Gardner et al., 2011) that alter soil chemical and physical properties (Bais et al., 2006; Compant et al., 2011). Soil bearing endophytes are thought to initially infect host plants through abrasions caused by microbial or nematode phytopathogens (McCully, 2001) from where they can quickly spread to intercellular spaces in the root (Chi et al., 2005). Then microbes can form biofilms, microcolonies, or aggregates. Attachment is mediated by several factors including outer polysaccharides and proteins (Assmus et al., 1995; Burdman et al., 1999). Competent root colonizers were defined as those who are capable of invading specific plant tissue, such as vascular tissue, spreading throughout the plant by manipulating plant metabolism, and maintaining a harmonious balance with the plant host, even when they are present in high density (Hardoim et al., 2008). In-situ image analysis of green fluorescent protein tagged rhizobia confirmed the ascending migration of endophytes from the rhizoplane into rice plants up to the stem base, leaf sheath and leaves where they developed high populations (Chi et al., 2005). Thus, it was suggested that there is tissue specificity for fungal endophytes (Kumar and Hyde, 2004).

There is evidence for the transfer of endophytes to progeny via seeds (Barrow et al., 1997, 2004; Schardl et al., 2004). Bacillus spp. endophytes of flower and fruit, can colonize seed cell wall as confirmed via FISH (fluorescent in-situ hybridization). Specific endophytic taxa in flowers and berries derive from the environment horizontally via roots, maternally via seeds or paternally via wind pollination (Compant et al., 2011). Association of fungal and bacterial endophytes was confirmed to occur at the level of seed embryo (Lucero et al., 2011).

In this study, we evaluated plant endophytes and soil microbial diversity for Atriplex spp. in arid regions, with a focus on the soil-plant continuums. Such evidence would support our hypothesis that environmentally recruited microorganisms might establish endophytically in plant tissues, including seeds, and would offer support to future tests assessing that some environmentally recruited endophytes might be then available for vertical transfer via seeds.

\section{Materials and methods}

\subsection{Study area and sampling method}

In order to test our hypothesis, we selected three arid regions with similar environments, i.e. the Jornada LTER (Long Term Ecological Research) site in New Mexico, USA, the research station of Jordan University of Science and Technology (JUST) and the Khanasri research station in Badia region of Jordan. Atriplex spp. plant units were used as model systems in this study. Atriplex is a genus with considerable phenotypic variability. The genus comprises many halophytes, and is widely distributed across arid and saline environments worldwide and valued for its restoration potential (Plummer, 1984; Khair et al., 2000). Fitness of Atriplex spp. is attributed to several factors, including its association with endophytes (Lucero et al., 2006; Barrow et al., 2008). In this study, Atriplex spp. plants in Jornada LTER, JUST and Khanasri regions were selected. Jornada LTER site is dominated by shrubs, such as Prosopis glandulosa and Atriplex spp. The region is characterized by arid climate with low annual precipitation of $23 \mathrm{~cm}$ 
(mainly falling during the summer monsoon, July to October). Average summer temperature is as high as $36^{\circ} \mathrm{C}$ during June (Peters et al., 2010). Both regions in Jordan are also characterized by arid climate. Khanasri research station only receives about $15 \mathrm{~cm}$ of annual precipitation, while the research station of JUST receives $59 \mathrm{~cm}$. Both Jordanian regions have mainly silty clay soils and are dominated by shrubs of Atriplex spp. and Salsola spp. (Shakhatreh et al., 2001; Al-Bakri et al., 2008; Alzweiri et al., 2011). In this study, plants sampled from the regions of Jordan were identified as Atriplex halimus, while those from Jornada LTER site of USA were identified as Atriplex canescens var. canescens. Mature plants of Atriplex canescens have simple, linear-spatulate to narrowly oblong and alternate leaves with a length of 1.3 to $5.0 \mathrm{~cm}$, and the heights of the species range from 0.3 to $2.4 \mathrm{~m}$. Flowering of this species is dioecious or monoecious (Welsh et al., 2003; Ogle et al., 2012). Atriplex halimus (also known as Mediterranean saltbush), with the maximum height of $3 \mathrm{~m}$, has deltoid-orbicular or lanceolate leaves covered with vesicular hairs. Flowering of this species is monoecious (Walker et al., 2014). Voucher specimens of the two Atriplex species were deposited in the Range Science Herbarium of the US Department of Agriculture in Las Cruces, New Mexico, USA.

Field sampling in Jornada LTER site was carried out at the end of November 2009 from undisturbed area in the northern Chihuahua (NC) Desert (32 $\left.{ }^{\circ} 40^{\prime} 17^{\prime \prime N}, 106^{\circ} 43^{\prime} 05^{\prime \prime} \mathrm{W}\right)$. Similar sampling was also conducted at the end of November 2010 in two arid regions of Jordan: one was an undisturbed (non-grazed) area located at the research station of JUST (32²9'23"N, 3559'22"E) and the other was a grazed area located at the Khanasri research station (32 $\left.24^{\prime} 02^{\prime \prime} \mathrm{N}, 36^{\circ} 08^{\prime} 02^{\prime \prime} \mathrm{E}\right)$. Each sampling site had three replicated sampling units. Each unit was centred on a four-wing saltbush (Atriplex spp.) plant and included the area with a radius of $1 \mathrm{~m}$. Soil samples included rhizosphere and non-rhizosphere soils. At each site, non-rhizosphere soils were sampled at depths of 0.5 (crust), $0.5-5$ and $5-25 \mathrm{~cm}$. Soil sampling was carried out at 30 and $60 \mathrm{~cm}$ away from the central Atriplex spp. plant along three equally spaced, radial transects. Thus, there were a total of 9 replicates for each non-rhizosphere sample type per sampling site. Rhizosphere soil samples (soil loosely adhering to roots) were collected for each central Atriplex spp. shrub (three samples per site). All the soil samples were air dried, passed through a 3-mm sieve and stored at $-20^{\circ} \mathrm{C}$ in tightly sealed tubes until to be used.

Plant tissues were also sampled from each replicated sampling unit of each site. Mature seeds were randomly selected and newly growing leaves were collected from the very top of every Atriplex spp. plant. Fifteen to twenty leaves or seeds were collected from each plant. Plant tissues were individually surface disinfested (Chi et al., 2005) by soaking in a 1:100 solution of Zerotol $^{\mathrm{TM}}$ (Biosafe Systems, LLC, Connecticut, USA), and spun at low speed for $10 \mathrm{~min}$. Then they were transferred to a flask containing $70 \%$ ethanol and spun again at low speed for another 5 min. Tissues were then washed 3 times in sterile distilled water by soaking for $3 \mathrm{~min}$. Disinfected tissues were stored at $-20^{\circ} \mathrm{C}$ in sealed centrifuge tubes until to be used. To verify the quality of the disinfections, aliquots of randomly selected final rinse liquids were plated on potato dextrose agar (PDA). Since no growth was observed after $48 \mathrm{~h}$ of incubation at room temperature (about $22^{\circ} \mathrm{C}$ ), this confirmed the lack of any cross-sample contamination between soil and plant tissues.

\subsection{DNA extraction}

DNA from each sample was extracted separately. Plant samples were ground under liquid nitrogen with sterile mortar and pestle, and immediately transferred into bead tubes for DNA extraction and optimization provided with the PowerPlant ${ }^{\mathrm{TM}}$ DNA isolation kits. Soil DNA was extracted with the PowerSoil ${ }^{\mathrm{TM}}$ DNA extraction kit following the manufacturer's protocol (MoBio Laboratories, Inc., Carlsbad, California, USA). Isolated DNA was stored at $-20^{\circ} \mathrm{C}$ and used within one month. Backup DNA aliquots were stored at $-80^{\circ} \mathrm{C}$. Randomly selected aliquots of the extracted DNA samples were quantified on a NanoDrop spectrophotometer to ensure that sufficient genomic yields were obtained.

\subsection{Pyrosequencing}

A DNA-yield weighted composite sample for each sample type per each site was sent for 
sequencing (27 composite samples in total). We analyzed bacterial diversity for both plants and soils using bacterial tag-encoded FLX amplicon pyrosequencing (bTEFAP) based on the Titanium sequencing platform (Research and Testing Laboratory, Lubbock, TX, USA). Amplification was carried out with the Gray28F (5'-GAGTTTGATCNTGGCTCAG-3') and Gray519R (5'-GTNTTACNGCGGCKGCTG-3') primers (Ishak et al., 2011; Lucero et al., 2011). For the fungal tag-encoded FLX amplicon pyrosequencing (fTEFAP) analysis, the fungal rDNA internal transcribed spacer (ITS) regions were directly amplified with a set of primers designed to exclude Atriplex DNA (Lucero et al., 2011), ENDOITSF (5'-AAGGTCTCCGTAGGTGAAC-3') and ENDOITSR (5'-GTATCCCTACCTGATCCGAG-3').

\subsection{Phylogenetic analyses}

Raw sequences from bTEFAP and fTEFAP were depleted of barcodes and primers, then short sequences of $<300$ bp were removed, sequences with ambiguous base calls were removed, and sequences with homopolymer runs exceeding 6 bp were also removed. Sequences were then denoised and chimeras removed. All these data quality control steps were performed at the Research and Testing Laboratory, Inc., Lubbock TX, USA. Sequencing reads of $>300$ bp were then clustered to consensus sequences at 96\% identity using the CD-HIT-454 4.5.3 (Niu et al., 2010). Consensus sequences that represented sequences present in at least one soil compartment (soil crust, subsurface non-rhizosphere soil or rhizosphere soil) and two plant compartments (seeds and leaves) were selected for alignment. For putative identification of bacteria up to genus level, we used the RDP Naive Bayesian rRNA Classifier Version 2.6 with a 50\% confidence threshold (Wang et al., 2007). The lower threshold was employed to minimize the proportion of sequences perceived as novel. It should be noted that this is a statistical confidence threshold but not a sequence identity value. For the fungal dataset, a nucleotide sequences similarity search via BLASTn was carried out against the CAMERA fungal nucleotide sequence database using standard NCBI parameters (Sun et al., 2010). Blastn results were visualized in the MEtaGenome ANalyzer (MEGAN version 4.70.4) against the NCBI taxonomy employing a least common ancestor (LCA) filter with one sequence support per taxon, given that the dataset included only consensus sequences, and 0.3 minimum complexity for non-repetitive sequences. Consensus sequences representative of these across the soil-plant continuums were then aligned, separately for each sampling site, by ClustalW 2.0 software (Larkin et al., 2007). This alignment did not assume identity but was more adept at comparing similar sequences across the sampled compartments. A maximum likelihood (ML) tree was prepared for each alignment by PhyML, with a Jukes-Cantor substitution model and bootstrap (100 cycles) optimized for tree topology, branch length and base substitution rate (Guindon and Gascuel, 2003). PhyML trees were visualized in SplitsTree software using a Neighbor-Joining method (Huson and Bryant, 2006). We employed Venn diagrams (Oliveros, 2007) to visualize the shared distribution of bacterial or fungal operational taxonomic units (OTUs) between seeds, leaves, and rhizosphere and non-rhizosphere soils. To simplify these diagrams, we combined all unique non-rhizosphere soil OTUs into a single dataset (data are not shown). We also performed the SIMPER (similarity percentage) analyses using the PAST software (version 3.08) (Hammer et al., 2001) to rank the shared bacterial and fungal taxa common for both soil and plant samples. Pyrosequencing data is publicly available in sequence depository NCBI Sequence Read Archive (SRA) (Project accession number: PRJNA243088 and study accession number: SRP041003).

\section{Results and discussion}

\subsection{Overview of sequence data}

A total of 90,829 pyrosequencing bacterial 16s rDNA reads and 125,142 pyrosequencing fungal ITS reads, quality controlled, were identified in this study (data are not shown). They were grouped into 1,764, 1,873 and 2,138 bacterial OTUs for Khanasri, JUST and Jornada LTER regions, respectively, and 513, 637 and 461 fungal OTUs for Khanasri, JUST and Jornada LTER regions, respectively. Fifteen bacterial OTUs and fourteen fungal OTUs were ubiquitous across 
all the study regions (Fig. 1). Of the total bacterial OTUs, $0.17 \%$ in Khanasri research station, $0.16 \%$ in research station of JUST and $0.42 \%$ in Jornada LTER site were commonly found across all the soil-plant continuums. However, if sharing between soil and plant compartments considered all OTUs present in at least one soil compartment and one plant compartment, then the shared values for bacteria increase to $10.88 \%$, 6.57\% and $6.17 \%$ in Khanasri, JUST and Jornada LTER regions, respectively (Fig. 1). For fungal OTUs, a similar pattern was observed. No fungal OTUs in Khanasri research station and only $1.56 \%$ in research station of JUST and $0.86 \%$ in Jornada LTER site were shared across all the soil-plant continuums. The lack of ubiquitous OTUs across all the tested soil-plant continuums in Khanasri research station might be just a statistical peculiarity of the sampling and DNA handling protocol. If sharing between soil and plant compartments was considered for OTUs present in at least one soil compartment and one plant compartment, then the shared values for fungi increase to $12.08 \%, 11.14 \%$ and $17.35 \%$ for Khanasri, JUST and Jornada LTER regions, respectively (Fig. 1). Arthrobacter, Sporosarcina and Cladosporium, as identified by RDP classification (Wang et al., 2007), were distributed across all the soil-plant continuums. Their average pairwise, sequence based, identity was $93.2 \%$ for Arthrobacter spp., 94.0\% for Sporosarcina spp. and 97.0\% for Cladosporium spp.
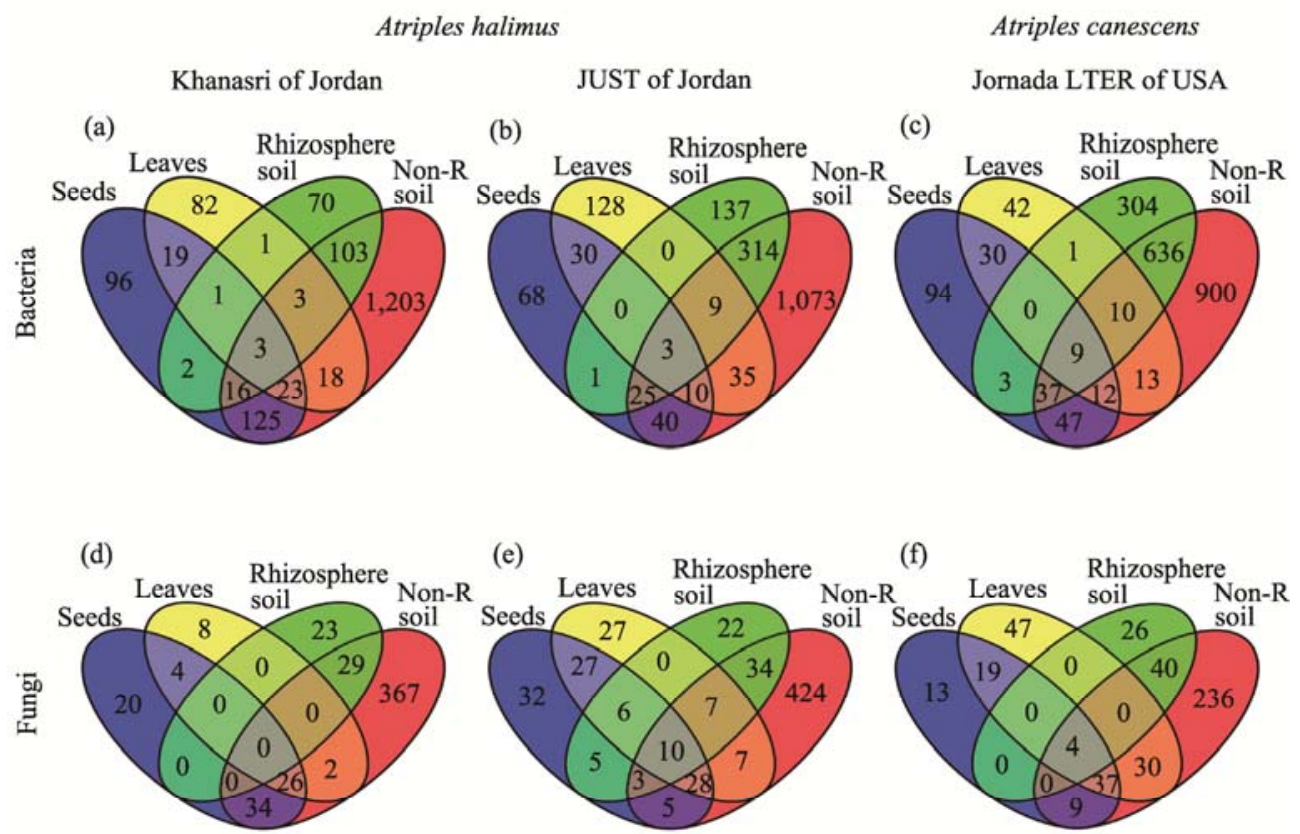

Fig. 1 Distribution of bacterial and fungal OTUs (operational taxonomic units) across the tested soil-plant continuums for Atriplex halimus and Atriplex canescens in Khanasri and JUST (Jordan University of Science and Technology) regions of Jordan and Jornada LTER (Long Term Ecological Research) site of USA. For each site, OTUs for the 6 types of non-rhizosphere soils were integrated into a single representative set. Non-R soil, non-rhizosphere soil.

\subsection{Examination of bacterial communities}

Putative Blastococcus spp. (Geodermatophilaceae) and an OTU assigned to Bogoriellaceae were found in plant compartments, and rhizosphere and non-rhizosphere soil compartments for Khanasri research station (Fig. 2). Arthrobacter spp. (Micrococcaceae) and Nocardioides spp. (Nocardioidaceae) were the most common bacteria across the soil-plant continuums for the research station of JUST (Fig. 3). Sphingomonadaceae, putative Oxalobacteraceae, Enterobacteriaceae, Planococcaceae, and Carnobacteriaceae were found in plant compartments and soils for both the Khanasri and JUST research stations (Figs. 2 and 3). Bacterial amplicons, commonly found across all the soil-plant continuums and isolated in Jornada LTER site (Fig. 4), 


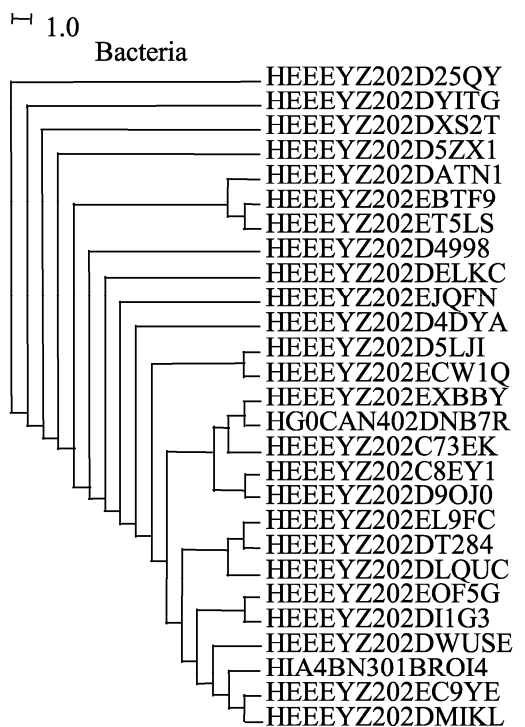

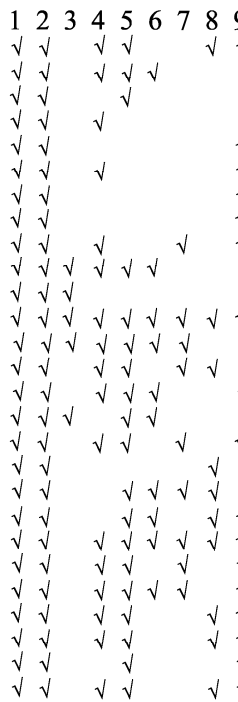

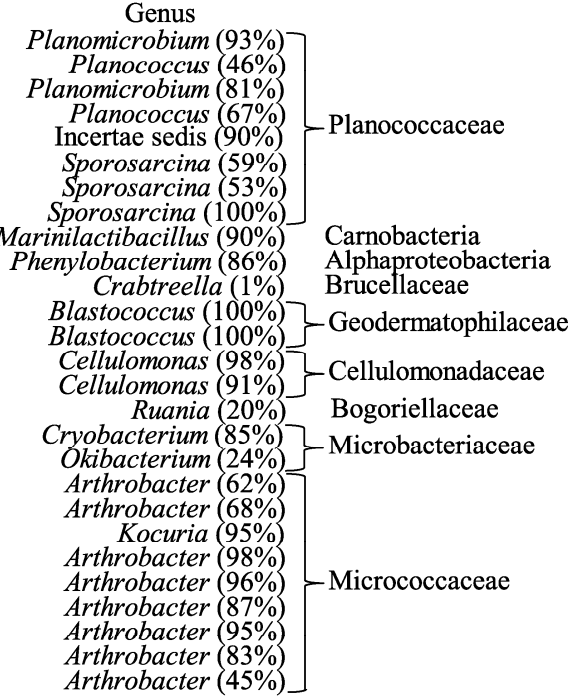

Frang

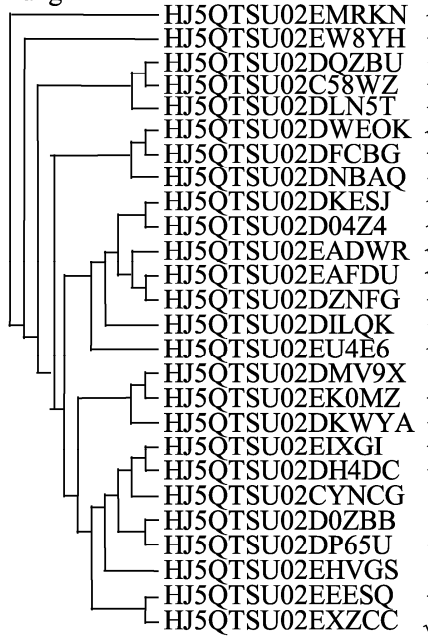

1233456789

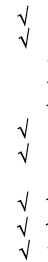

\section{Genus}

Phaeotheca $(100 \%)$

Phaeotheca $(100 \%)\}$ Capnodiales (order)

Pleospora (57\%)

Pleospora $57 \%$ - Pleosporaceae

Pleospora $(57 \%$

Cladosporium $78 \%$

Cladosporium $78 \%$

Unclassified fungi $(72 \%$

Cladosporium $(80 \%)$

Cladosporium $(80 \%)$

Cladosporium $52 \%$

Cladosporium $(52 \%)$

Cladosporium $(50 \%)$

Cladosporium $52 \%$

Cladosporium $(50 \%)$

Cladosporium $(100 \%$

Cladosporium $(100 \%)$

Cladosporium $100 \%$

Davidiella $(100 \%)$

Cladosporium $100 \%$

Cladosporium $100 \%$

Cladosporium $(100 \%)$

Davidiella $(100 \%)$

Cladosporium $(100 \%$

Cladosporium $100 \%$

Cladosporium (100\%)

Fig. 2 Phylogeny of the bacterial and fungal consensus sequences in the soil-plant continuums of Khanasri research station, Jordan. 1, seeds; 2, leaves; 3 , rhizosphere soil; 4, soil crust at $30 \mathrm{~cm}$ distance from the plant; 5 , soil crust at $60 \mathrm{~cm}$ distance from the plant; 6, non-rhizosphere soil at depth of $0.5-5.0 \mathrm{~cm}$ and at $30 \mathrm{~cm}$ distance from the plant; 7, non-rhizosphere soil at depth of $5-30 \mathrm{~cm}$ and at $30 \mathrm{~cm}$ distance from the plant; 8, non-rhizosphere soil at depth of $0.5-5.0 \mathrm{~cm}$ and at $60 \mathrm{~cm}$ distance from the plant; 9, non-rhizosphere soil at depth of 5-30 cm and at $60 \mathrm{~cm}$ distance from the plant. Phaeoteca spp., a mitosporic Ascomycota, is classified in the Capnodiales order.

were related to Geodermatophilaceae, Planococcaceae, Pseudonocardiaceae, Chitinophagaceae, Methylobacteriaceae and Caulobacteraceae.

Bacterial amplicons, commonly found across all the soil-plant continuums and isolated in Jornada LTER site, were putatively related to Geodermatophilaceae, Pseudonocardiaceae, Chitinophagaceae, Planococcaceae, Methylobacteriaceae and Caulobacteraceae. However, Deinococcaceae, Oxalobacteraceae, Cellulomonadaceae, Rhodobacteraceae and Brucellaceae families were only found in plant tissues and non-rhizosphere soils (Fig. 4).

JUST and Jornada LTER regions produced indicator signatures that were found across all the tested soil-plant continuums. The dynamic distributions of members of Proteobacteria and Actinobacteria along all the soil-plant continuums in this study were in agreement with those in earlier findings (Chi et al., 2005) on the ascending endophytic migration of soil rhizobia, e.g. from roots of Japonica rice (Oryza sativa L.) through the stem base, then developing high 
populations in leaves and eventually persisting into the reproductive phases of development. A number of Proteobacteria and Actinobacteria previously isolated from the roots of Atriplex halimus overlapped with those isolated from the under-canopy soil and bulk soil of the same plant (Kaplan et al., 2013). These observations might indicate the plant recruitment of these bacteria from soils (Chi et al., 2005; Bulgarelli et al., 2013) via the rooting system. Microbiota retrieved from the surface-sterilized seeds and stems of 14 genotypes of corn cultivars, landraces and ancestors (teosinte) revealed that seed endosphere profiles were distinct for each pedoclimatic regions, supporting the hypothesis that some endophytes might be environmentally recruited. Thus, the initial inoculum in the surrounding soil biome likely determined the endophyte community structure in the context of genetics of plant species (Bulgarelli et al., 2013). Intuitively, there is a possibility that some of these endophytes might have been recruited from air and aerosols. However, soil is a stronger inoculum source since the typical bacterial titer in the air is significantly lower than that in the soil (Whitman et al., 1998). Nonetheless, this should not eliminate the aerial route of transmission for aerosol-associated bacteria (Fahlgren et al., 2010).

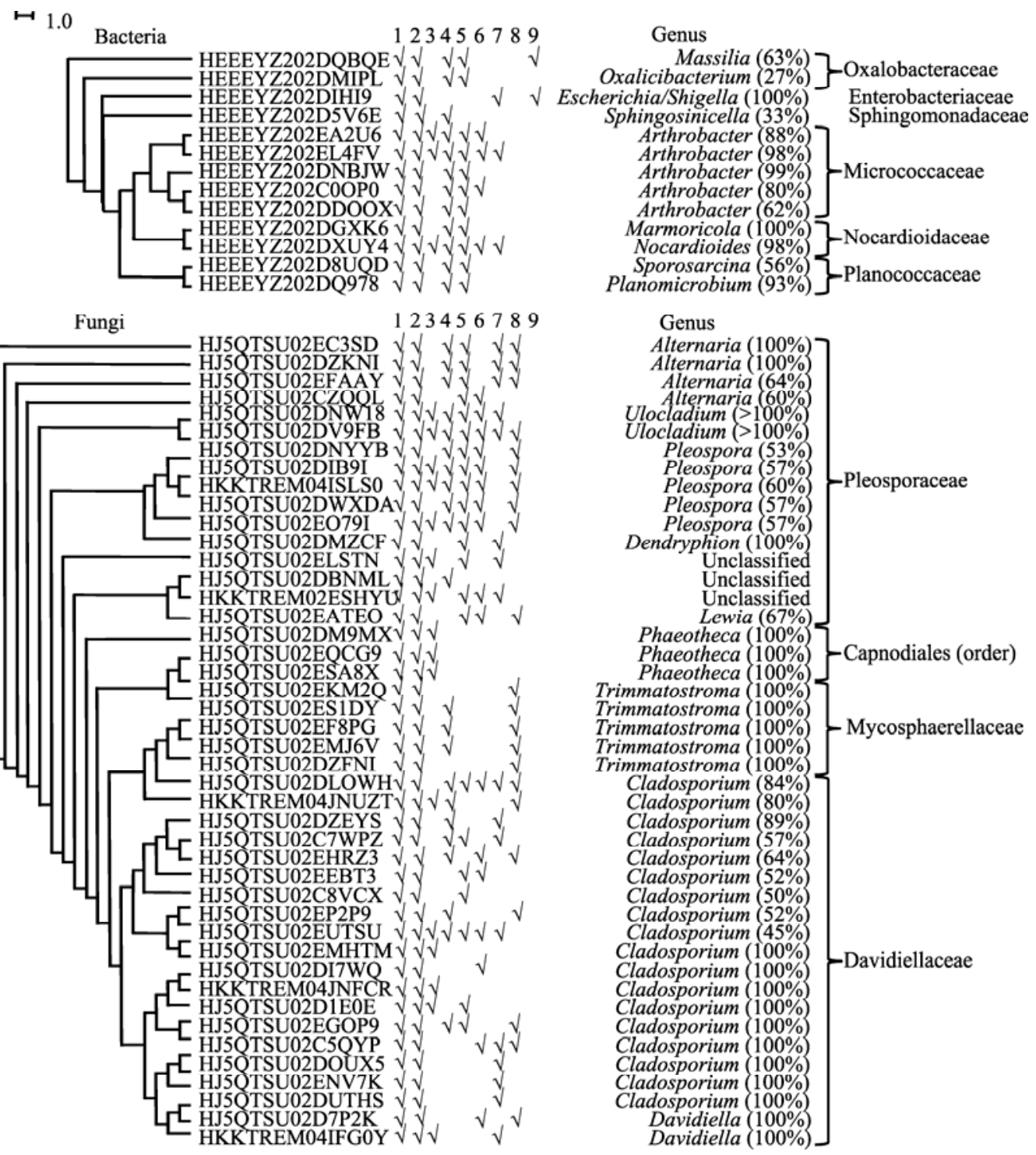

Fig. 3 Phylogeny of the bacterial and fungal consensus sequences in the soil-plant continuums of the research station of JUST, Jordan. Numbers 1-9 in the figure follow the same description as indicated in Fig. 2. Phaeoteca spp., a mitosporic Ascomycota, is classified in the Capnodiales order. 
$\mapsto 1.0$

Bacteria



HIA4BN301BIFT2

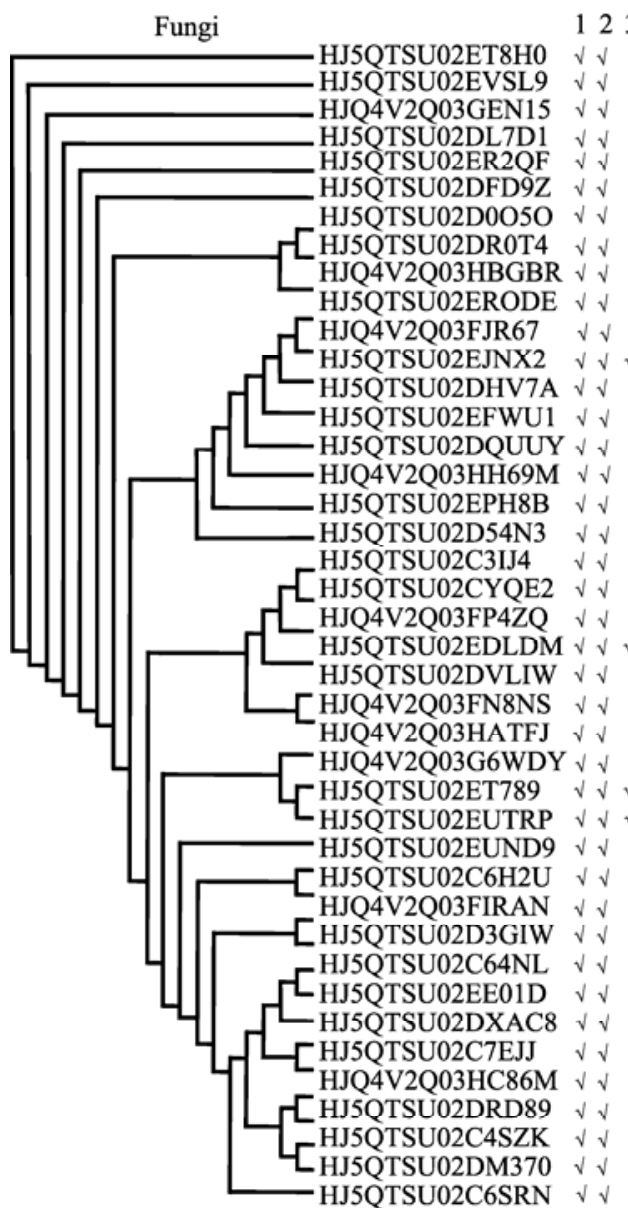

123456789
Genus

Geodermatophilus $(48 \%)$
Geodermatophilus $(88 \%)$ -Geodermatophilace Arthrobacter $(47 \%)$ Micrococcaceae

Modestobacter (42\%)

Modestobacter (43\%) -Geodermatophilace

Deinococcus $(100 \%)$

Deinococcus $(100 \%)$ Deinococcaceae

Arthrobacter (90\%)

Cellulomonas $(100 \%) \quad$ Cellulomonadaceae

Pseudonocardia (97\%) Pseudonocardiaceae

Flavisolibacter $(39 \%)$

Terrimonas $(77 \%)$-Chitinophagaceae

Sporosarcina $(63 \%)$

Planococcaceae

Phenylobacterium $(91 \%) \quad$ Caulobacteraceae

Microvirga $(95 \%)$

Microvirga $(68 \%) \quad-$ Methylobacteriacea

Microvirga (38\%)

Rubellimicrobium (94\%) Rhodobacteraceae

Herbaspirillum $(25 \%) \quad$ Oxalobacteraceae

Crabtreella (2\%) -Brucelaceae

Genus

Aureobasidium (100\%)

Aureobasidium (75\%) -Dothioraceae

Aureobasidium (100\%)

Aureobasidium (100\%)

Unclassified fungal endophyte

Cladosporium $(60 \%)$

Cladosporium (100\%) Davidiellaceae

Cladosporium (52\%)

Leptospherulina $(50 \%)$

Phoma (67\%)

Phoma (75\%)

Phoma (67\%)

Phoma (40\%)

Phoma (67\%)

Leptosphaeria $(100 \%)$ Unclassified

Dendryphion (100\%) Unclassified

Dendryphion (50\%) Unclassified

Unclassified

Dendryphion (50\%) Unclassified

Alternaria $(100 \%)$

Alternaria $(100 \%)$

Alternaria $(64 \%)$

Alternaria $(60 \%)$

Alternaria (100\%) -Pleosporaceae

Alternaria (100\%)

Alternaria $(60 \%)$

Alternaria $(100 \%)$

Alternaria (100\%)

Alternaria (100\%)

Alternaria (100\%)

Unclassified

Alternaria (84\%)

Alternaria (100\%)

Alternaria $(100 \%)$

Alternaria (100\%)

Alternaria $(64 \%)$

Fig. 4 Phylogeny of the bacterial and fungal consensus sequences in the soil-plant continuums of Jornada LTER site, USA. Numbers 1-9 in the figure follow the same description as indicated in Fig. 2.

\subsection{Examination of fungal communities}

Ascomycota fungal consensus sequences belonging to Pleosporaceae and Davidiellaceae were identified across all the soil-plant continuums in JUST research station of Jordan (Fig. 3). However, in Khanasri research station of Jordan, Davidiellaceae members were only commonly 
found in plant compartments and non-rhizosphere soils (Fig. 2). In Jornada LTER site, Phoma (Didymellaceae) and Alternaria (Pleosporaceae) were found across all the tested soil-plant continuums (Fig. 4). For both Jornada LTER and JUST regions, Dothioraceae, Leptosphaeriaceae and Pleosporaceae were only commonly found in plant compartments and non-rhizosphere soils (Figs. 3 and 4).

Most of the Ascomycota fungi detected in plant tissues were also found in the rhizosphere and non-rhizosphere soils (Figs. 2-4). Previous reports suggested that the endophytic life style may protect these microbes from the harsh desert conditions and function as mycorrhiza for promoting plant growth (Kaplan et al., 2013). Seventeen Ascomycota described previously for micropropagated Atriplex canescens that were strictly obtained from excised embryos (Lucero et al., 2011) included taxa that were also found across the soil-plant continuums (Dothioraceae, Cladosporium, Alternaria, Davidiellaceae and Didymellaceae) in our study area. Since these have also been found in the soils, it may suggest that these endophytes are either environmentally recruited to become seedborne endophytes and/or are inoculated into soils by germinating seeds.

\subsection{Similarity analyses of bacterial and fungal communities for the soil-plant continuums}

In this study, we performed SIMPER analysis to rank the contribution of shared taxa to the differences in microbial communities among different regions (Table 1). The results showed that Arthrobacter and Sporosarcina were the most common genera and contributed most to the dissimilarities among different sampling sites, accounting for $32.85 \%$ of the observed dissimilarity in bacterial communities. Moreover, Cladosporium and Alternaria genera occupied $58.00 \%$ of the observed dissimilarity in associated fungal communities.

The overall dissimilarity for microbial communities was $86.13 \%$ and $81.71 \%$ for bacteria and fungi, respectively. SIMPER analysis showed that two bacterial genera (Arthrobacter and Sporosarcina) and two fungal genera (Cladosporium and Alternaria) had high dissimilarities in all sampling sites, indicating their possible importance in the soil-plant continuums.

A Pearson correlation analysis that considered both presence and abundance for the specific bacterial OTUs of soil-plant continuums showed that, there was a high similarity between the two regions of Jordan ( $r=0.78$ for Khanasri and JUST), but there was no correlation between these two regions and the Jornada LTER site of USA ( $r=0.02$ for Khanasri and Jornada LTER, and $r=-0.02$ for JUST and Jornada LTER). Similar patterns were also found for the specific fungi of soil-plant continuums ( $r=0.94$ for Khanasri and JUST, $r=-0.07$ for Khanasri and Jornada LTER, and $r=0.02$ for JUST and Jornada LTER). Moreover, a matching shared species analysis that only considered presence (Jaccard classic index, SJ as calculated in EstimateS) (Colwell, 2013) showed that there was high dissimilarity for bacterial taxonomic diversity for all comparison regions (SJ=0.20 for Khanasri and JUST, SJ=0.24 for Khanasri and Jornada LTER, and SJ=0.13 for JUST and Jornada LTER). Similarity patterns were also noted for fungi among different regions (SJ=0.50 for Khanasri and JUST, SJ=0.27 for Khanasri and Jornada LTER, and SJ=0.11 for JUST and Jornada LTER). The goal of this study was to evaluate the diversity of endophytes across the soil-plant continuum communities in different arid regions. Both of the obvious differences in endophyte populations between USA and Jordan, and the high similarity among the Jordanian communities might point to a biogeographical separation hypothesis. Although the plant species in this study derived from the same genus, they were also distinct between USA and Jordan, so any further inference would be speculative.

\subsection{Endophytes continuity along the soil-plant continuum and their putative significance}

Seedborne endophyte consortia previously reported for Atriplex species (Lucero et al., 2011) included isolates associated with improved seedling vigor and plant nutrient uptake, suggesting a role in overall plant fitness (Barrow et al., 1997; Barrow and Osuna, 2002; Lucero et al., 2006). Our study firstly aimed to find evidence for taxonomic continuity of endophytes across the soil-plant continuums in arid regions. Identification of putative OTUs was a secondary goal. We examined microbial populations in the soil-plant continuums, from seeds, to leaves and to rhizosphere and non-rhizosphere soils, to identify putatively common indicator rDNA signatures. 
The sharing of endophytic microorganisms between environmental sinks and plant tissues, including seeds, raised the question of functional continuity between vegetative tissue and plant-soil-microbial communities. Functions were not experimentally attributed to the taxa identified here, across the soil-plant continuum. However, previous knowledge suggested a wide range of potential functions from putative diazotrophes (Oxalobacteraceae and Enterobacteriaceae) (Martínez-Falcón et al., 2011), antifungal producers (Pseudonocardiaceae and Chitinophagaceae) (Basilio et al., 2003; Miranda et al., 2013), to methane-oxidizers and phenolic compounds degraders (Methylobacteriaceae and Caulobacteraceae) (Hinrichs et al., 2000; Flynn et al., 2013).

Table 1 SIMPER (similarity percentage) analysis for identifying the contribution of bacterial and fungal taxa to the dissimilarity of microbial communities and mean abundance of microbial communities for the three study regions

\begin{tabular}{|c|c|c|c|c|c|c|c|}
\hline \multirow[b]{2}{*}{ Genus } & \multirow[b]{2}{*}{ Family } & \multirow[b]{2}{*}{$\begin{array}{c}\text { Average } \\
\text { dissimilarity } \\
(\%)\end{array}$} & \multirow[b]{2}{*}{$\begin{array}{l}\text { Percentage } \\
\text { (\%) }\end{array}$} & \multirow[b]{2}{*}{$\begin{array}{c}\text { Cumulative } \\
\text { percentage } \\
(\%)\end{array}$} & \multicolumn{3}{|c|}{ Mean abundance } \\
\hline & & & & & $\begin{array}{c}\text { JUST } \\
\text { of } \\
\text { Jordan } \\
\end{array}$ & $\begin{array}{c}\text { Khanasri } \\
\text { of } \\
\text { Jordan }\end{array}$ & $\begin{array}{c}\text { Jornada } \\
\text { LTER } \\
\text { of USA }\end{array}$ \\
\hline \multicolumn{8}{|l|}{ Bacteria } \\
\hline Arthrobacter & Micrococcineae & 21.12 & 24.52 & 24.52 & 5 & 7 & 1 \\
\hline Sporosarcina & Planococcaceae & 7.18 & 8.32 & 32.85 & 1 & 3 & 1 \\
\hline Planomicrobium & Planococcaceae & 5.24 & 6.09 & 38.93 & 1 & 2 & 0 \\
\hline Deinococcus & Deinococcaceae & 5.21 & 6.05 & 44.98 & 0 & 0 & 2 \\
\hline Microvirga & Methylobacteriaceae & 5.21 & 6.05 & 51.03 & 0 & 0 & 2 \\
\hline Cellulomonas & Cellulomonadaceae & 5.13 & 5.96 & 56.98 & 0 & 2 & 1 \\
\hline Blastococcus & Geodermatophilaceae & 3.51 & 4.07 & 61.06 & 0 & 2 & 0 \\
\hline Phenylobacterium & Alphaproteobacteria & 3.38 & 3.92 & 64.98 & 0 & 1 & 1 \\
\hline Massilia & Oxalobacteraceae & 2.75 & 3.19 & 68.16 & 1 & 0 & 0 \\
\hline Marmoricola & Enterobacteriaceae & 2.75 & 3.19 & 71.35 & 1 & 0 & 0 \\
\hline Escherichia/Shigella & Enterobacteriaceae & 2.75 & 3.19 & 74.54 & 1 & 0 & 0 \\
\hline Nocardioides & Nocardioidaceae & 2.75 & 3.19 & 77.73 & 1 & 0 & 0 \\
\hline Terrimonas & Chitinophagaceae & 2.60 & 3.02 & 80.75 & 0 & 0 & 1 \\
\hline Geodermatophilus & Geodermatophilaceae & 2.60 & 3.02 & 83.77 & 0 & 0 & 1 \\
\hline Pseudonocardia & Pseudonocardiaceae & 2.60 & 3.02 & 86.80 & 0 & 0 & 1 \\
\hline Rubellimicrobium & Rhodobacteraceae & 2.60 & 3.02 & 89.82 & 0 & 0 & 1 \\
\hline Incertae sedis & Planococcaceae & 1.75 & 2.04 & 91.86 & 0 & 1 & 0 \\
\hline Planococcus & Planococcaceae & 1.75 & 2.04 & 93.89 & 0 & 1 & 0 \\
\hline Marinilactibacillus & Carnobacteriaceae & 1.75 & 2.04 & 95.93 & 0 & 1 & 0 \\
\hline Cryobacterium & Microbacteriaceae & 1.75 & 2.04 & 97.96 & 0 & 1 & 0 \\
\hline Kocuria & Micrococcineae & 1.75 & 2.04 & 100.00 & 0 & 1 & 0 \\
\hline \multicolumn{8}{|l|}{ Fungi } \\
\hline Cladosporium & Davidiellaceae & 28.92 & 35.39 & 35.39 & 18 & 18 & 3 \\
\hline Alternaria & Pleosporaceae & 18.48 & 22.62 & 58.00 & 4 & 0 & 16 \\
\hline Pleospora & Pleosporaceae & 6.63 & 8.11 & 66.12 & 5 & 3 & 0 \\
\hline Phoma & Didymellaceae & 5.22 & 6.39 & 72.51 & 0 & 0 & 5 \\
\hline Trimmatostroma & Mycosphaerellaceae & 4.52 & 5.53 & 78.05 & 5 & 0 & 0 \\
\hline Aureobasidium & Dothioraceae & 4.18 & 5.11 & 83.16 & 0 & 0 & 4 \\
\hline Phaeotheca & Capnodiales (order) & 4.12 & 5.04 & 88.20 & 3 & 2 & 0 \\
\hline Dendryphion & Pleosporaceae & 3.58 & 4.38 & 92.57 & 1 & 0 & 3 \\
\hline Davidiella & Davidiellaceae & 3.21 & 3.93 & 96.51 & 2 & 2 & 0 \\
\hline Ulocladium & Pleosporaceae & 1.81 & 2.21 & 98.72 & 2 & 0 & 0 \\
\hline Leptosphaeria & Leptosphaeriaceae & 1.04 & 1.28 & 100.00 & 0 & 0 & 1 \\
\hline
\end{tabular}

Note: The average dissimilarity was calculated using the Bray-Curtis method. JUST, Jordan University of Science and Technology; LTER, Long Term Ecological Research. Phaeoteca spp., a mitosporic Ascomycota, is classified in the Capnodiales order.

\section{Conclusion}

This study shows that at all sampling sites, certain bacterial and fungal indicator sequences, and thus possibly organisms, were commonly found across the soil and endophytic plant 
compartments. While not comprehensively verifiable here, biogeographic specificity or plant species specificity of endophytes may be nevertheless hypothesized. Vertical transfer of environmentally recruited organisms via seeds is thus a possibility. Inoculation of soils by endophytes from germinating seeds is also a possibility. The present investigation emphasizes the need and value for further investigation of biota shared across the soil-plant continuum to verify these hypotheses and identify any associated functionalities crucial for assessing ecological risks in endophyte assisted restoration. Results of our research may present an avenue for biologically sound options for the reclamation of degraded arid landscapes, based on the understanding of the significance of these endophytes present along soil-plant continuum.

\section{Acknowledgments}

The authors thank Dr. Veena UNNITHAN and Dr. Mark SEGER for technical support with the molecular analyses and bioinformatics. Thanks are also extended to Dr. Ameen GHARAYBEH and Mr. Mohammad GHARAYBEH from Jordan University of Science and Technology for assistance with field sampling.

\section{References}

Al-Bakri J T, Ajlouni M, Abu-Zanat M. 2008. Incorporating land use mapping and participation in Jordan: An approach to sustainable management of two mountainous areas. Mountain Research and Development, 28(1): 49-57.

Alzweiri M, Al Sarhan A, Mansi K, et al. 2011. Ethnopharmacological survey of medicinal herbs in Jordan, the Northern Badia region. Journal of Ethnopharmacology, 137(1): 27-35.

Assmus B, Hutzler P, Kirchhof G, et al. 1995. In situ localization of Azospirillum brasilense in the rhizosphere of wheat with fluorescently labeled, rRNA-targeted oligonucleotide probes and scanning confocal laser microscopy. Applied and Environmental Microbiology, 61(3): 1013-1019.

Bais H P, Weir T L, Perry L G, et al. 2006. The role of root exudates in rhizosphere interactions with plants and other organisms. Annual Review of Plant Biology, 57: 233-266.

Barrow J R, Havstad K M, Hubstenberger J, et al. 1997. Seed-borne fungal endophytes on fourwing saltbush, Atriplex canescens. Arid Soil Research Rehabilitation, 11(4): 307-314.

Barrow J R, Osuna P. 2002. Phosphorus solubilization and uptake by dark septate fungi in fourwing saltbush, Atriplex canescens (Pursh) Nutt. Journal of Arid Environments, 51(3): 449-459.

Barrow J R, Osuna-Avila P, Reyes-Vera I. 2004. Fungal endophytes intrinsically associated with micropropagated plants regenerated from native Bouteloua eriopoda Torr. and Atriplex canescens (Pursh) NUTT. In Vitro Cellular \& Developmental Biology-Plant, 40(6): 608-612.

Barrow J, Lucero M, Reyes-Vera I, et al. 2008. Do symbiotic microbes have a role in regulating plant performance and response to stress? Communicative \& Integrative Biology, 1(1): 69-93.

Basilio A, González I, Vicente M F, et al. 2003. Patterns of antimicrobial activities from soil actinomycetes isolated under different conditions of $\mathrm{pH}$ and salinity. Journal of Applied Microbiology, 95(4): 814-823.

Bulgarelli D, Schlaeppi K, Spaepen S, et al. 2013. Structure and functions of the bacterial microbiota of plants. Annual Review of Plant Biology, 64: 807-838.

Burdman S, Jurkevitch E, Schwartsburd B, et al. 1999. Involvement of outer-membrane proteins in the aggregation of Azospirillum brasilense. Microbiology, 145(5): 1145-1152.

Chi F, Shen S H, Cheng H P, et al. 2005. Ascending migration of endophytic rhizobia, from roots to leaves, inside rice plants and assessment of benefits to rice growth physiology. Applied Environmental Microbiology, 71(11): 7271-7278.

Colwell R K. 2013. EstimateS: Statistical Estimation of Species Richness and Shared Species from Samples (version 9.1.0). User's Guide and Application. http://purl.oclc.org/estimates.

Compant S, Mitter B, Colli-Mull J, et al. 2011. Endophytes of grapevine flowers, berries, and seeds: identification of cultivable bacteria, comparison with other plant parts, and visualization of niches of colonization. Microbial Ecology, 62(1): $188-197$.

Dalling J W, Davis A S, Schutte B J, et al. 2011. Seed survival in soil: interacting effects of predation, dormancy and the soil microbial community. Journal of Ecology, 99(1): 89-95.

Doty S L. 2008. Enhancing phytoremediation through the use of transgenics and endophytes. New Phytologist, 179(2): 318-333.

Fahlgren C, Hagstrom $\AA$, Nilsson D, et al. 2010. Annual variations in the diversity, viability, and origin of airborne bacteria. Applied and Environmental Microbiology, 76(9): 3015-3025. 
Flynn T M, Sanford R A, Ryu H, et al. 2013. Functional microbial diversity explains groundwater chemistry in a pristine aquifer. BMC Microbiology, 13(1): 146.

Gallery R E, Dalling J W, Arnold A E. 2007. Diversity, host affinity, and distribution of seed-infecting fungi: A case study with Cecropia. Ecology, 88(3): 582-588.

Gardner T, Acosta-Martinez V, Senwo Z, et al. 2011. Soil rhizosphere microbial communities and enzyme activities under organic farming in Alabama. Diversity, 3(3): 308-328.

Guindon S, Gascuel O. 2003. A simple, fast, and accurate algorithm to estimate large phylogenies by maximum likelihood. Systematic Biology, 52(5): 696-704.

Hammer Ø, Harper D A T, Ryan P D. 2001. PAST: Paleontological statistics software package for education and data analysis. Palaeontologia Electronica, 4(1): 1-9.

Hardoim P R, Van Overbeek L S, Van Elsas J D. 2008. Properties of bacterial endophytes and their proposed role in plant growth. Trends in Microbiology, 16(10): 463-471.

Hinrichs K U, Summons R E, Orphan V, et al. 2000. Molecular and isotopic analysis of anaerobic methane-oxidizing communities in marine sediments. Organic Geochemistry, 31(12): 1685-1701.

Huson D H, Bryant D. 2006. Application of phylogenetic networks in evolutionary studies. Molecular Biology and Evolution, 23(2): 254-267.

Ishak H D, Plowes R, Sen R, et al. 2011. Bacterial diversity in Solenopsis invicta and Solenopsis geminata ant colonies characterized by $16 \mathrm{~S}$ amplicon 454 Pyrosequencing. Microbial Ecology, 61(4): 821-831.

Johnston-Monje D, Raizada M N. 2012. Conservation and diversity of seed associated endophytes in Zea across boundaries of evolution, ethnography, and ecology. PLoS ONE, 6(6): e20396, doi:10.1371/journal.pone.0020396.

Kaplan D, Maymon M, Agapakis C M, et al. 2013. A survey of the microbial community in the rhizosphere of two dominant shrubs of the Negev Desert highlands, Zygophyllum dumosum (Zygophyllaceae) and Atriplex halimus (Amaranthaceae) using cultivation-dependent and cultivation-independent methods. American Journal of Botany, 100(9): 1713-1725.

Khair M, El-Shatnawi J, Mohawesh Y M. 2000. Seasonal chemical composition of saltbush in semiarid grasslands of Jordan. Journal of Range Management, 53(2): 211-214.

Kumar D, Hyde K D. 2004. Biodiversity and tissue-recurrence of endophytic fungi in Tripterygium wilfordii. Fungal Diversity, 17: 69-90.

Larkin M A, Blackshields G, Brown N P, et al. 2007. Clustal W and Clustal X version 2.0. Bioinformatics, 23(21): 2947-2948.

Leuchtmann A. 1993. Systematics, distribution, and host specificity of grass endophytes. Journal of Natural Toxins, 1(3): 150-162.

Lucero M E, Barrow J R, Osuna P, et al. 2006. Plant-fungal interactions in arid and semiarid ecosystems: large scale impacts from microscale processes. Journal of Arid Environments, 65(2): 276-284.

Lucero M E, Barrow J R, Osuna P, et al. 2008. Enhancing native grass productivity by co-cultivating with endophyte-laden calli. Rangeland Ecology and Management, 61(1): 124-130.

Lucero M E, Unc A, Cooke P, et al. 2011. Endophyte microbiome diversity in micropropagated Atriplex canescens and Atriplex torreyi var griffithsii. PLoS ONE, 6(3): e17693, doi: 10.1371/journal.pone.0017693.

Lynch J M, Whipps J M. 1991. Substrate flow in the rhizosphere. In: Keister D L, Cregan P B. The Rhizosphere and Plant Growth. Netherlands: Springer, 14: 15-24.

Martínez-Falcón A P, Durbán A, Latorre A, et al. 2011. Bacteria associated with Copestylum (Diptera, Syrphidae) larvae and their cactus host Isolatocereus dumortieri. PLoS ONE, 6(11): e27443, doi: 10.1371/journal.pone.0027443.

McCully M E. 2001. Niches for bacterial endophytes in crop plants: a plant biologist's view. Australian Journal of Plant Physiology, 28(9): 983-990.

Miranda L N, Hutchison K, Grossman A R, et al. 2013. Diversity and abundance of the bacterial community of the red macroalga Porphyra umbilicalis: did bacterial farmers produce macroalgae? PLoS ONE, 8(3): e58269, doi: 10.1371/journal.pone.0058269.

Niu B F, Fu L M, Sun S L, et al. 2010. Artificial and natural duplicates in pyrosequencing reads of metagenomic data. Bioinformatics, 11: 187.

Ogle D, St. John L, Tilley D. 2012. Plant guide for fourwing saltbush Atriplex canescens (Pursh) Nutt. In: USDA-Natural Resources Conservation Service, Aberdeen, ID Plant Materials Center. 83210-0296.

Oliveros J, Venny C. 2007. An interactive tool for comparing lists with Venn Diagrams. [2015-04-15]. http://bioinfogp.cnb.csic.es/tools/venny/index.html.

Peters D P C, Herrick J E, Monger H C, et al. 2010. Soil-vegetation-climate interactions in arid landscapes: effects of the North American monsoon on grass recruitment. Journal of Arid Environments, 74(5): 618-623. 
Plummer M. 1984. Considerations in selecting chenopod species for range seedings. In: Tiedemann A R, McArthur E D, Stutz H C, et al. Proceedings Symposium on the Biology of Atriplex and Related Chenopods. U.S. Department of Agriculture, Forest Service, Intermountain Forest and Range Experiment Station, 183-186.

Saikkonen K, Wäli P, Helander M, et al. 2004. Evolution of endophyte plant symbioses. Trends in Plant Sciences, 9(6): 275-280.

Schardl C L, Leuchtmann A, Spiering M J. 2004. Symbioses of grasses with seedborne fungal endophytes. Annual Review of Plant Physiology, 55: 315-340.

Shakhatreh Y, Kafawin O, Ceccarelli S, et al. 2001. Selection of barley lines for drought tolerance in low-rainfall areas. Journal of Agronomy and Crop Science, 186(2): 119-127.

Sun S L, Chen J, Li W Z, et al. 2010. Community cyberinfrastructure for advanced microbial ecology research and analysis: the CAMERA resource. Nucleic Acids Research, 39(suppl 1): D546-D551.

Walker D J, Lutts S, Sánchez-Garcíac M, et al. 2014. Atriplex halimus L.: Its biology and uses. Journal of Arid Environments, 100-101: 111-121.

Wang Q, Garrity G M, Tiedje J M, et al. 2007. Naïve Bayesian classifier for rapid assignment of rRNA sequences into the new bacterial taxonomy. Applied and Environmental Microbiology, 73(16): 5261-5267.

Welsh S L, Atwood N D, Goodrich S, et al. 2003. A Utah Flora ( $3^{\text {rd }}$ ed.). Provo, UT: Brigham Young University, 1-912.

Whitman W B, Coleman D C, Wiebe W J, 1998. Prokaryotes: the unseen majority. Proceedings of the National Academy of Sciences of the United States of America, 95(12): 6578-6583.

Wilson D. 1995. Endophyte: the evolution of a term, and clarification of its use and definition. Oikos, 73(2): $274-276$. 